\title{
Biomarkers in psychiatry: how close are we?
}

\author{
Firas Kobeissy ${ }^{1 *}$, Ali Alawieh², Stefania Mondello ${ }^{3}$, Rose-Mary Boustany ${ }^{2}$ and Mark S. Gold ${ }^{1}$ \\ ${ }^{1}$ Division of Addiction Medicine, Department of Psychiatry, Center for Neuroproteomics and Biomarkers Research, University of Florida, Gainesville, FL, USA \\ ${ }^{2}$ Department of Biochemistry, College of Medicine, American University of Beirut, Beirut, Lebanon \\ ${ }^{3}$ Department of Neuroscience, University of Messina, Messina, Italy \\ *Correspondence: firasko@gmail.com
}

Edited by:

Sulie L. Chang, Seton Hall University, USA

Reviewed by:

Sulie L. Chang, Seton Hall University, USA

\section{A commentary on}

Systems biology, bioinformatics and biomarkers in neuropsychiatry by Alawieh, A., Zaraket, F., Li, J., Mondello, S., Nokkari, A., Razafsha, M., et al. (2012). Front. Neurosci. 6:187. doi: 10.3389/fnins.2012.00187

Until recently, there has been an ongoing worldwide quest in search for disease-specific molecular biomarkers in medicine. These biological molecules can allow for: a reliable and accurate disease diagnosis and prognosis, better understanding of pathogenesis and pathophysiological mechanisms, and for predicting disease progression and monitoring therapy. Furthermore, biomarkers can provide major opportunities for drug target identification which can ultimately translate into new therapeutic strategies with disease-modifying effects. Notably, all of these conditions are inherent characteristics of neuropsychiatric diseases.

Biomarker research has achieved great success in various clinical fields such as cardiovascular disease, hepatic disorders, neurotrauma leading to key markers including the discovery of troponin as marker for myocardial infarction, and 14-3-3 protein for Creuzfolt-Jacob Disease, S100//UCH-L1/ $\alpha$ II-spectrin for brain trauma (Hayes et al., 2011; Kobeissy et al., 2011; Mondello et al., 2011). However, in psychiatry this field is still lagging since no putative biomarker has yet made its way into clinical application (Schulenborg et al., 2006; Lescuyer et al., 2007).

Biological psychiatry research has been introduced as an attempt to draw psychiatry back to its biological roots in order to improve injury mechanisms and disease processes and its components. It has been well-understood today in clinical medicine that no promising accurate and definite disease diagnosis, therapy, and prognosis can be established without drawing back the clinical manifestation of the disease. Therefore, biological psychiatry is now focusing on the use of all available advanced molecular techniques that can allow for biomarker detection assisted by the afore-employed imaging and analysis techniques. Such approaches include the utilization of high throughput omics approaches such as: epigenetics, genomics, proteomics, lipidomics, and metabolomics studies (Robeva, 2010; Westerhoff, 2011). In addition, these methodologies rely on sophisticated computational-multi disciplinary field of systems biology utilizing advanced bioinformatics processing tools that can interpret the high throughput molecular omics data relevant to neuropsychiatric research. Among the ultimate aims of such discipline is the identification of novel sensitive and disease-specific biomarker(s).

The promise that systems biology can lead a progress in biological psychiatry returns to the very complex nature of psychiatric disorders. Such disorders involve multifactorial genetic and environmental interactions together with the dynamic nature of protein alterations affecting both cellular as well as structural changes on the neuronal levels. Therefore, assessing psychiatric disorders cannot be targeted at a single behavioral or cellular level but rather would require a holistic global approach that can assess different components of such disorders
(Fang and Casadevall, 2011; Westerhoff, 2011). This can lead the inquiry into the roots of such disorders and identify new diagnostic and assessment biomarkers. However, the need for biomarker discovery and the implementation of systems biology techniques is not just because of the complexity of the disease. It is also an attempt to surmount the available diagnostic techniques such as DSM IV and ICD-10 that involve "subjective" checklist analysis of signs and symptoms of these diseases that causes frustration among most psychiatric practitioners (Linden, 2012; Tretter and GebickeHaerter, 2012).

Having been said, there has been a pronounced worldwide joint effort in the advancing of biomarker studies that is evident by the surge of research and review articles focusing on the application of systems biology, bioinformatics, and biomarkers in neuropsychiatry. These studies have included the use of high-throughput genomic, epigenetics, proteomic, metabolomics, and other-bioinformatic computational algorithms tools as well as the use of animal models, in vitro and in vivo tissue cultures and in silico models. These techniques have been applied on different aspects of neuropsychiatric disorders spanning: drug abuse, eating disorders, and other psychiatric disorders involving schizophrenia, bipolar disorder, and major depressive disorder etc. (Kobeissy et al., 2008; Avena, 2011).

The application of these techniques has provided several disease models of psychiatric diseases (Tretter and GebickeHaerter, 2012) that have moved research and therapy forward as with the dopamine agonist model of schizophrenia that 
we reviewed in a separate publication (Alawieh et al., 2012). However, success reported by using such techniques is still in its infancy due to the aforementioned complexity of psychiatric diseases as well as for other reasons. These include, on one hand, the limitations associated with these techniques coupled with "mindset" related to scientists and researchers that emphasizes on data discovery rather than data analysis and validation. This resulted in massive amount of data-majorly non-replicable and non-validated-with very low biological significance and clinical impact (Kraemer et al., 2002; Staner, 2006; Martins-DeSouza et al., 2011). Therefore, there is now an uprising need for the integrative and predictive analysis as well as validation of the available data collected to infer the biological significance relevant to psychiatry.

Finally, the field of biomarker discovery in psychiatry, taking advantage of systems biology approach and the available bioinformatics tools, is believed to yield several advantages including early diagnosis that is critical to psychiatric diseases and accurate criteria for disease, diagnosis, classification, and stratification. It can also allow for advanced personalized therapy and can act, if appropriately, validated as surrogate end points that can eliminate several limitations and greatly advance clinical research (Biomarkers Definitions Working Group, 2001; Zhang et al., 2010).

\section{REFERENCES}

Alawieh, A., Zaraket, F., Li, J., Mondello, S. Nokkari, A., Razafsha, M., et al. (2012). Systems biology, bioinformatics and biomarkers in neuropsychiatry. Front. Neurosci. 6:187. doi: 10.3389/fnins.2012.00187

Avena, N. M. (2011). Food and addiction: implications and relevance to eating disorders and obesity. Curr. Drug Abuse Rev. 4, 131-132.

Biomarkers Definitions Working Group. (2001) Biomarkers and surrogate endpoints: preferred definitions and conceptual framework. Clin. Pharmacol. Ther. 69, 89-95.

Fang, F. C., and Casadevall, A. (2011). Reductionistic and holistic science. Infect. Immun. 79, 1401-1404.

Hayes, R. L., Mondello, S., and Wang, K. (2011). Glial fibrillary acidic protein: a promising biomarker in pediatric brain injury. Pediatr. Crit. Care Med. 12, 603-604.

Kobeissy, F. H., Guingab-Cagmat, J. D., Razafsha, M., O'Steen, L., Zhang, Z., Hayes, R. L., et al. (2011). Leveraging biomarker platforms and systems biology for rehabilomics and biologics effectiveness research. $P M R 3$, S139-S147.

Kobeissy, F. H., Warren, M. W., Ottens, A. K., Sadasivan, S., Zhang, Z., Gold, M. S., et al. (2008). Psychoproteomic analysis of rat cortex following acute methamphetamine exposure. J. Proteome Res. 7, 1971-1983.

Kraemer, H. C., Schultz, S. K., and Arndt, S. (2002). Biomarkers in psychiatry: methodological issues. Am. J. Geriatr. Psychiatry 10, 653-659.

Lescuyer, P., Hochstrasser, D., and Rabilloud, T. (2007). How shall we use the proteomics toolbox for biomarker discovery? J. Proteome Res. 6 , 3371-3376.

Linden, D. E. (2012). The challenges and promise of neuroimaging in psychiatry. Neuron 73, 8-22.

Martins-De-Souza, D., Guest, P. C., VanattouSaifoudine, N., Harris, L. W., and Bahn, S. (2011). Proteomic technologies for biomarker studies in psychiatry: advances and needs. Int. Rev. Neurobiol. 101, 65-94.
Mondello, S., Linnet, A., Buki, A., Robicsek, S., Gabrielli, A., Tepas, J., et al. (2011). Clinical utility of serum levels of ubiquitin C-terminal hydrolase as a biomarker for severe traumatic brain injury. Neurosurgery 70, 666-675.

Robeva, R. (2010). Systems biology - old concepts, new science, new challenges. Front. Psychiatry 1:1. doi: 10.3389/fpsyt.2010.00001

Schulenborg, T., Schmidt, O., Van Hall, A., Meyer, H. E., Hamacher, M., and Marcus, K. (2006). Proteomics in neurodegeneration-disease driven approaches. J. Neural Transm. 113, 1055-1073.

Staner, L. (2006). Surrogate outcomes in neurology, psychiatry, and psychopharmacology. Dialogues Clin. Neurosci. 8, 345-352.

Tretter, F., and Gebicke-Haerter, P. J. (2012). Systems biology in psychiatric research: from complex data sets over wiring diagrams to computer simulations. Methods Mol. Biol. 829, 567-592.

Westerhoff, H. V. (2011). Systems biology left and right. Methods Enzymol. 500, 3-11.

Zhang, Z., Larner, S. F., Kobeissy, F., Hayes, R. L., and Wang, K. K. (2010). Systems biology and theranostic approach to drug discovery and development to treat traumatic brain injury. Methods Mol. Biol. 662, 317-329.

Received: 13 December 2012; accepted: 17 December 2012; published online: 07 January 2013.

Citation: Kobeissy F, Alawieh A, Mondello S, Boustany $R-M$ and Gold MS (2013) Biomarkers in psychiatry: how close are we? Front. Psychiatry 3:114. doi: 10.3389/ fpsyt.2012.00114

This article was submitted to Frontiers in Molecular Psychiatry, a specialty of Frontiers in Psychiatry.

Copyright (c) 2013 Kobeissy, Alawieh, Mondello, Boustany and Gold. This is an open-access article distributed under the terms of the Creative Commons Attribution License, which permits use, distribution and reproduction in other forums, provided the original authors and source are credited and subject to any copyright notices concerning any third-party graphics etc. 